\title{
CONSERVAÇÃO DE SEMENTES DE IPÊ-AMARELO (Tabebuia chrysotricha (Mart. ex A. DC.) Standl.) EM FUNÇÃO DO TEOR DE ÁGUA DAS SEMENTES E DA TEMPERATURA DO ARMAZENAMENTO
}

\author{
LEILA MARTINS ${ }^{1}$, ANTÔNIO AUGUSTO DO LAGO², WILSON ROBERTO MARQUES SALES ${ }^{3}$.
}

\begin{abstract}
RESUMO - O ipê-amarelo (Tabebuia chrysotricha (Mart. ex A. DC.) Standl.) é uma espécie arbórea de alto valor ecológico, econômico e paisagístico. Suas sementes apresentam variação significativa na qualidade durante o armazenamento, o que representa dificuldades no desenvolvimento de técnicas de propagação. O objetivo desta pesquisa foi estudar o comportamento fisiológico das sementes de ipê-amarelo durante o armazenamento através de variações no teor de água das sementes e na temperatura do ambiente. Frutos colhidos manualmente de plantas matrizes foram colocados para secagem e posterior extração das sementes. Primeiramente, foi determinado o teor de água inicial do lote e, paralelamente, obtida a porção com o maior teor de água a ser estudado (21,1\%). Em seguida, as sementes remanescentes foram submetidas à secagem, em secador com circulação forçada de ar a $28 \pm 2{ }^{\circ} \mathrm{C}$, por diferentes períodos, obtendo-se teores de água de $15,9,13,6,11,9$ e 8,5\%. As porções correspondentes aos diferentes teores de água foram armazenadas a $-12,10$ e $20^{\circ} \mathrm{C}$. No início e a cada 30 dias até 270 dias de armazenamento, as sementes foram submetidas às avaliações de teor de água, germinação, emergência, velocidade de emergência e comprimento da parte aérea. O mais alto e o mais baixo teor de água estudado $(21,1 \%$ e $8,5 \%)$ são prejudiciais à qualidade fisiológica das sementes mesmo à temperatura extremamente baixa $\left(-12^{\circ} \mathrm{C}\right)$ de armazenamento. A conservação das sementes de ipê-amarelo (Tabebuia chrysotricha) é favorecida quando armazenadas com teor de água em torno de $11,9 \%$ nas temperaturas de 10 e $-12^{\circ} \mathrm{C}$.
\end{abstract}

Termos para indexação: Tabebuia chrysotricha, propagação, temperatura de armazenamento, qualidade fisiológica.

\section{PRESERVATION OF Tabebuia chrysotricha SEEDS AS A FUNCTION OF SEED WATER CONTENT AND STORAGE TEMPERATURE}

\begin{abstract}
Tabebuia chrysotricha (Mart. ex A. DC.) Standl. is a tree species of high ecological, economical and landscape value. Its seeds present significant variation in quality during storage, which hampers the development of propagation techniques. The objective of this research was, through variations in seed water content and environment temperature, to study the physiological
\end{abstract}

Submetido em 04/04/2008. Aceito para publicação em 21/10/2008.

${ }^{1}$ Laboratório Central de Sementes e Mudas, Departamento de Sementes Mudas e Matrizes - CATI. Caixa Postal 962, CEP 13070-178, Campinas, SP, Brasil, email: leila@cati.sp.gov.br. Bolsista do CNPq.

${ }^{2}$ Instituto Agronômico de Campinas/APTA. Caixa Postal 28, CEP 13001-
970, Campinas, SP, Brasil, email: aalago@iac.sp.gov.br

${ }^{3}$ Núcleo de Produção de Mudas de Pederneiras, Departamento de Sementes Mudas e Matrizes - CATI. Estrada Velha de Jaú, s/nº, Bairro Duas Passagens, Pederneiras, SP, Brasil, email: npm.pederneiras@cati.sp.gov.br 
performance of Tabebuia chrysotricha seeds during storage. Fruits were hand-collected from mother plants and placed under shade for a short time to allow easy extraction by hand. Firstly, the initial moisture content of the whole seed lot was determined, and a portion of it was drawn so that to represent the control treatment, with the highest moisture level to be studied $(21,1 \%)$. Following that, the remaining seeds were dried in a forced air dryer at $28 \pm 2^{\circ} \mathrm{C}$, for different periods, in order to obtain water contents of $15,9,13,6,11,9$, and $8,5 \%$. The portions, corresponding to the different moisture degrees were stored at temperatures of $-12,10$ and $20^{\circ} \mathrm{C}$. Initially and at 30 day intervals until 270 days of storage, seeds were evaluated in relation to water content, germination, emergence, speed of emergence, and length of aerial seedling parts. The highest and the lowest moisture contents studied ( 21,1 and $8,5 \%)$ were detrimental to the physiological quality of the seeds even at the extremely low storage temperature of $-12^{\circ} \mathrm{C}$. The preservation of Tabebuia chrysotricha seeds, with water content between $8,5 \%$ and $22,1 \%$, is favored by the water content of $11,9 \%$ at the temperatures of 10 and $-12^{\circ} \mathrm{C}$.

Index terms:: Tabebuia chrisotricha, propagation, storage condition, physiological quality.

\section{INTRODUÇÃO}

O conhecimento das características ecofisiológicas das sementes de espécies nativas, visando à produção de mudas, dentre outros objetivos, para recuperar ou enriquecer áreas degradadas, é importante para a manutenção da biodiversidade das espécies. Os tecnologistas de sementes florestais encontram ainda dificuldades no estabelecimento de técnicas de produção, manipulação e avaliação da qualidade das sementes, devido à grande variação biomorfológica encontrada nos frutos e sementes das diferentes espécies.

Como a produção dessas sementes é limitada no tempo é de fundamental importância o estudo de condições de armazenamento adequadas à manutenção da capacidade germinativa, visando à semeadura na época favorável (Oliveira et al., 2006). Adicionalmente, a produção irregular de frutos de um ano para outro pode prejudicar a formação de estoques reguladores e, também, proporcionar lotes com quantidade insuficiente de sementes até mesmo para os testes de avaliação da qualidade (Aguiar et al., 1993). Além disso, há falta de informações para a definição de procedimentos, capazes de maximizar o período de conservação das sementes, específicos para cada espécie.

Tabebuia chrysotricha (Mart. ex A. DC.) Standl.), popularmente conhecida como ipê-amarelo, pau-d'arcoamarelo, ipê-amarelo-cascudo, entre outros nomes populares, é uma Bignoniaceae de porte arbóreo, alcançando altura de 4 a $10 \mathrm{~m}$, com características de planta heliófila e decídua (Lorenzi, 2002). Sua ocorrência estende-se desde o Estado do Espírito Santo até o de Santa Catarina na floresta pluvial atlântica; é comum na vegetação secundária localizada sobre solos bem drenados de encostas, capoeiras e capoeirões e apresenta valor ecológico, paisagístico e econômico (Lorenzi, 2002).

As sementes de espécies do gênero Tabebuia apresentam forte variação na qualidade durante o armazenamento (Carvalho, 1994) e, além disso, possuem período de viabilidade curto, o que representam dificuldades no estabelecimento de técnicas de cultivo para o reflorestamento de áreas degradadas, além de limitar sua dispersão natural (Pinto et al., 1986).

Segundo Roberts (1973), sementes ortodoxas com teor de água inferior a $5 \%$ podem ser armazenadas à baixas temperaturas, por longo período, sem prejudicar seu potencial germinativo. Foi o que constataram Figliolia et al. (1986/88) para sementes de Tabebuia vellosoi Toledo, cujas sementes liofilizadas e armazenadas em condições de ambiente, com $7,5 \%$ de água, conservaram integralmente a germinação até 240 dias, apresentando ainda elevada capacidade germinativa até 420 dias de armazenamento. Melo e Eira (1995), também observaram que sementes de ipê-branco, com $9 \%$ de teor de água, mantiveram o poder germinativo durante dois anos armazenadas a $-20^{\circ} \mathrm{C}$. Por outro lado, Degan et al. (2001) observaram que sementes de Tabebuia roseo-alba (Ridl.) (ipê-branco) liofilizadas e com 3,7\% de teor de água tiveram significativa redução de germinabilidade depois de 60 dias de armazenamento em ambiente de laboratório (sem controle da temperatura e da umidade relativa do ar) e depois de 120 dias em câmara seca (sem controle da temperatura e com umidade relativa do ar em torno de $40 \%$ ). Carvalho et al. 
(1976) verificaram em sementes de ipê-amarelo (Tabebuia chrysotricha (Mart. ex A. DC.) Standl.), germinação de aproximadamente $80 \%$ após 150 dias de armazenamento em câmara seca (sem controle da temperatura e umidade relativa do ar em torno de $40 \%)$ e fria $\left(10^{\circ} \mathrm{C}\right.$ e $86 \%$ de umidade relativa do ar). No entanto, Natale e Carvalho (1983) constataram perda total do potencial fisiológico de sementes de ipê-roxo após 150 dias de armazenamento (em ambiente normal de laboratório) e somente $2 \%$ de germinação aos 120 dias.

Kano et al. (1978) observaram que os ipês apresentam baixa longevidade das sementes, restringindo sua utilização em reflorestamento, pois, sementes com $81 \%$ de germinação inicial e $9,1 \%$ de teor de água, acondicionadas em embalagem permeável, apresentaram reduções progressivas da qualidade fisiológica durante o armazenamento até a perda total da capacidade germinativa aos 240 dias.

Portanto, observa-se que o teor de água das sementes e a temperatura do ambiente de armazenamento são fatores determinantes na conservação das sementes de ipê.

O objetivo desta pesquisa foi estudar o comportamento fisiológico de sementes de Tabebuia chrysotricha (Mart. ex A. DC.) Standl. (ipê-amarelo) com teores de água distintos em diferentes condições de armazenamento.

\section{MATERIAL E MÉTODOS}

A pesquisa foi realizada no Laboratório Central de Sementes e Mudas do Departamento de Sementes, Mudas e Matrizes (LCSM/DSMM) pertencente à Coordenadoria de Assistência Técnica Integral (CATI), em Campinas. As sementes de Tabebuia chrysotricha (Mart. ex A. DC.) Standl. (ipê-amarelo) foram provenientes do Núcleo de Produção de Mudas de Pederneiras - DSMM/CATI.

Os frutos foram colhidos maduros, porém fechados, de oito plantas matrizes e colocados em ambiente sombreado de laboratório para secagem natural e posterior extração das sementes. Primeiramente, foi determinado o teor de água inicial do lote (BRASIL, 1992) e, paralelamente, obtida a amostra representativa do tratamento controle, com o maior teor de água a ser estudado $( \pm 22 \%)$. Em seguida, as sementes remanescentes foram submetidas à secagem em secador com circulação constante de ar a $28 \pm 2^{\circ} \mathrm{C}$, para a obtenção dos demais teores de água desejados $(16,13,11$ e $8 \%)$. Esses teores foram definidos no intuito de estudar os efeitos da conservação de sementes com teor de água muito alto (22\%), alto $(16 \%)$, intermediários $(13 \%$ e $11 \%)$ e baixo $(8 \%)$.
Os teores de água foram obtidos com acompanhamento da perda de massa das sementes durante a secagem. Para tanto, as amostras de sementes com massas iniciais previamente conhecidas foram acondicionadas em sacos de filó e distribuídas nas bandejas do secador para monitoramento da perda de massa a intervalos regulares. As massas finais das amostras, correspondentes a cada um dos teores de água desejados, foram calculados por meio da equação descrita

por Cromarty et al. (1985): $\quad M_{f}=M_{i} \frac{\left(100-U_{i}\right)}{\left(100-U_{f}\right)}$
sendo:

$\mathrm{M}_{\mathrm{f}}=$ massa da amostra ( $\mathrm{g}$ ) após a secagem;

$\mathrm{M}_{\mathrm{i}}=$ massa da amostra $(\mathrm{g})$ antes da secagem;

$\mathrm{U}_{\mathrm{i}}=$ grau de umidade (\%) antes da secagem;

$\mathrm{U}_{\mathrm{f}}=$ grau de umidade (\%) desejado após a secagem.

Durante esse processo e à medida em que os teores de água estavam próximos dos desejados, amostras foram retiradas, homogeneizadas e divididas em frações que, por sua vez, foram embaladas em sacos de polietileno $(0,14 \mathrm{~mm}$ de espessura) e mantidas a $20^{\circ} \mathrm{C} \pm 2{ }^{\circ} \mathrm{C}$, provisoriamente, até a obtenção de todos os teores de água.

As amostras, correspondentes aos diferentes teores de água, foram separadas para cada período de armazenamento, seladas e armazenadas em câmaras (geladeiras) reguladas a $-12^{\circ} \mathrm{C} \pm 2{ }^{\circ} \mathrm{C}, 10^{\circ} \mathrm{C} \pm 2{ }^{\circ} \mathrm{C}$ e $20^{\circ} \mathrm{C}$ $\pm 2^{\circ} \mathrm{C}$. Antes do armazenamento e após este aos 30,60 , $90,120,150,180,210,240$ e 270 dias as sementes foram submetidas às seguintes avaliações:

Teor de água: realizado a $105^{\circ} \mathrm{C} \pm 3^{\circ} \mathrm{C}$ por $24 \mathrm{~h}$, pelo método da estufa (BRASIL, 1992), em duas amostras de $1 \mathrm{~g}$ por tratamento. Os resultados obtidos, com base na massa úmida $(\mathrm{Bu})$, foram expressos em porcentagem.

Germinação: realizada utilizando-se 200 sementes (8 subamostras de 25 sementes) dentro de recipiente de vidro fechado com tampa, $25 \times 12 \times 5 \mathrm{~cm}$, mantidos a $25^{\circ} \mathrm{C}$ (Socolowski e Takaki, 2007) e $8 \mathrm{~h}$ diárias de luz. O substrato papel, foi umedecido com volume de água equivalente a três vezes a sua massa sem hidratação. As avaliações, feitas aos 7, 14, 21 e 28 dias após a instalação do teste, forneceram dados que foram expressos em porcentagem de plântulas normais (BRASIL, 1992).

Emergência das plântulas: foi avaliada semeando-se cinco subamostras de 20 sementes em caixas de plástico $(11 \times 11 \times 3,5 \mathrm{~cm})$ sem tampa, e substrato constituído de areia autoclavada a $120^{\circ} \mathrm{C}$ por $2 \mathrm{~h}$. As caixas foram distribuídas sobre bancada de alvenaria, em interior de laboratório e sob luz indireta, com temperatura e umidade relativa variáveis e sem o monitoramento destas. 
O substrato foi umedecido a aproximadamente $60 \%$ da capacidade de retenção de água (Marcos Filho et al., 1987). Foram consideradas plântulas normais as que após 30 dias da instalação do teste apresentaram a parte área exposta acima da superfície do substrato.

Velocidade de emergência das plântulas: obtida contando-se o número diário de plântulas emersas no teste de emergência, e calculando-se o índice (IVE) seguindo os procedimentos descritos por Marcos Filho et al. (1987).

Comprimento da parte aérea da plântula: avaliado aos 30 dias após a instalação do teste de emergência, quando foi tomado o comprimento $(\mathrm{cm})$ entre a região de transição da raiz com o hipocótilo e a região de inserção das folhas cotiledonares. Os dados médios foram obtidos pelo quociente entre o somatório das medidas registradas e o número de sementes utilizadas (Vanzolini et al., 2007).

O delineamento experimental foi o inteiramente casualizado, em esquema fatorial, envolvendo 5 tratamentos (teores de água) no início do armazenamento e 15 tratamentos ( 5 teores de água $\times 3$ temperaturas de armazenamento) para cada época de avaliação, durante o armazenamento. A comparação das médias foi realizada pelo teste de Tukey ao nível de 5\% de probabilidade. Os dados de germinação e emergência de plântulas foram transformados em arcoseno $\sqrt{\mathrm{x} / 100}$, em que $x$ referese à porcentagem de germinação ou de emergência de plântulas, mas nas tabelas são apresentadas as médias sem transformação (\%).

\section{RESULTADOS E DISCUSSÃO}

As avaliações realizadas antes do armazenamento (Tabela 1) demonstram proximidade entre os valores desejados para o teor de água das sementes e os valores experimentalmenteverificados. Considerando as avaliações fisiológicas realizadas (germinação, emergência, índice de velocidade de emergência e comprimento da parte aérea da plântula), os teores extremos de água (de 21,1 e $8,5 \%$ ) causaram efeitos negativos imediatos, pois as sementes desses tratamentos apresentaram no início do armazenamento, os percentuais de germinação e emergência de 11 e $8 \%$ e 10 e $6 \%$ respectivamente, os quais foram inferiores aos demais.

TABELA1. Teor deágua (TA, \%) desejado e obtido, germinação (G), emergência (E), índice de velocidade de emergência (IVE) e comprimento da parte aérea (CPA) obtidos a partir de sementes de Tabebuia chrysotricha (Mart. ex A. DC.) Standl. (ipê-amarelo) antes do armazenamento.

\begin{tabular}{|c|c|c|c|c|c|}
\hline \multicolumn{2}{|c|}{ TA $(\%)$} & \multirow{2}{*}{$\mathrm{G}(\%)$} & \multirow{2}{*}{$\mathrm{E}(\%)$} & \multirow{2}{*}{ IVE } & \multirow{2}{*}{$\mathrm{CPA}(\mathrm{cm}$} \\
\hline Desejado & Obtido & & & & \\
\hline 22 & 21,1 & $11 \mathrm{~b}$ & $8 \mathrm{~b}$ & $0,23 \mathrm{bc}$ & $0,16 \mathrm{c}$ \\
\hline 16 & 15,9 & $30 \mathrm{a}$ & $28 \mathrm{a}$ & $0,64 \mathrm{a}$ & $0,83 \mathrm{a}$ \\
\hline 13 & 13,6 & $24 \mathrm{a}$ & $28 \mathrm{a}$ & $0,54 \mathrm{a}$ & $0,73 \mathrm{ab}$ \\
\hline 11 & 11,9 & $24 \mathrm{a}$ & $22 \mathrm{a}$ & $0,46 \mathrm{ab}$ & $0,47 \mathrm{bc}$ \\
\hline 8 & 8,5 & $10 \mathrm{~b}$ & $6 \mathrm{~b}$ & $0,14 \mathrm{c}$ & $0,22 \mathrm{c}$ \\
\hline \multicolumn{2}{|c|}{$\mathrm{CV}(\%)$} & 15,71 & 12,49 & 11,03 & 8,31 \\
\hline
\end{tabular}

Médias seguidas de mesma letra na coluna não diferem entre si pelo teste de Tukey a 5\% de probabilidade. CV-coeficiente de variação experimental

Os teores de água das sementes apresentaram pequena variação durante o período experimental, quando comparados com os teores de água iniciais (Tabela 2), mantendo a identidade dos tratamentos com relação aos teores de água nas temperaturas consideradas. Dessa forma, a embalagem utilizada (sacos de polietileno com $0,14 \mathrm{~mm}$ de espessura) demonstrou eficiência na manutenção dos graus de umidade originalmente obtidos e permitiu confiabilidade nas comparações realizadas durante o armazenamento.

O teor de água de 8,5\% mostrou-se especialmente prejudicial visto que até mesmo no armazenamento a $-12^{\circ} \mathrm{C}$, as sementes apresentaram germinação e emergência de 
6,0\% após apenas 30 dias de armazenamento (Tabelas 3 e 4). Entretanto, Kageyama e Marques (1981) armazenaram sementes de ipê-dourado (Tabebuia sp) com teor de água ao redor de $8 \%$ em câmara seca (temperatura de aproximadamente $20^{\circ} \mathrm{C}$ e umidade relativa do ar de $45 \%$ ) e constataram que essa condição foi a que menos danos causou à viabilidade das sementes.

No conjunto de dados obtidos na avaliação da germinação (Tabela 3) observa-se que as combinações entre os teores de água e temperaturas de $13,6 \%$ a $-12^{\circ} \mathrm{C}$ e, principalmente, de $11,9 \%$ a $10^{\circ} \mathrm{C}$ e $-12^{\circ} \mathrm{C}$ favoreceram a manutenção do desempenho das sementes durante todo o período estudado. No entanto, o desempenho foi apenas aceitável em sementes armazenadas a $20^{\circ} \mathrm{C}$ com teores de água de $13,6 \%$ e $11,9 \%$ por 60 e 120 dias, respectivamente. Paralelamente, os teores de água mais elevados, ou seja, 22,1 e 15,9\%, nas temperaturas de 10 e $20^{\circ} \mathrm{C}$ causaram declínio rápido na germinação, particularmente após 60 dias, sugerindo ação fortemente prejudicial à conservação das sementes. Carvalho et al. (1976) obtiveram resultados favoráveis na conservação de sementes de ipê-amarelo (Tabebuia chrysotricha) em geladeira a $10 \pm 2{ }^{\circ} \mathrm{C}$ por aproximadamente 150 dias.

TABELA 2. Teor de água das sementes de Tabebuia chrysotricha (Mart. ex A. DC.) Standl. (ipê-amarelo) durante o armazenamento em diferentes condições de temperatura $\left(T,{ }^{\circ} \mathrm{C}\right)$ e de teor de água inicial das sementes (TAS).

\begin{tabular}{|c|c|c|c|c|c|c|c|c|c|c|}
\hline \multirow[b]{2}{*}{$\mathrm{T}$} & \multirow[b]{2}{*}{ TAS } & \multicolumn{9}{|c|}{ Período de armazenamento (dias) } \\
\hline & & 30 & 60 & 90 & 120 & 150 & 180 & 210 & 240 & 270 \\
\hline \multirow[t]{5}{*}{$10^{\circ} \mathrm{C}$} & $21,1 \%$ & 22,1 & 22,4 & 24,9 & 25,1 & 24,1 & 24,5 & 26,6 & 23,5 & 24,5 \\
\hline & $15,9 \%$ & 15,7 & 15,4 & 18,5 & 16,9 & 16,7 & 16,3 & 15,7 & 15,5 & 16,3 \\
\hline & $13,6 \%$ & 11,8 & 10,9 & 13,9 & 14,6 & 13,4 & 13,0 & 14,9 & 11,5 & 12,9 \\
\hline & $11,9 \%$ & 10,9 & 11,7 & 10,0 & 10,3 & 10,1 & 10,5 & 10,6 & 9,95 & 10,4 \\
\hline & $8,5 \%$ & 7,9 & 8,9 & 9,0 & 9,9 & 8,0 & 9,5 & 10,8 & 9,3 & 9,4 \\
\hline \multirow[t]{5}{*}{$20^{\circ} \mathrm{C}$} & $21,1 \%$ & 22,8 & 24,0 & 23,9 & 23,4 & 22,8 & 21,0 & 24,3 & 23,5 & 21,1 \\
\hline & $15,9 \%$ & 16,9 & 16,8 & 17,5 & 17,8 & 18,1 & 17,6 & 18,5 & 17,6 & 15,7 \\
\hline & $13,6 \%$ & 13,2 & 13,3 & 13,1 & 12,8 & 13,3 & 13,0 & 14,3 & 11,6 & 12,9 \\
\hline & $11,9 \%$ & 10,9 & 10,1 & 11,4 & 10,1 & 11,1 & 10,5 & 10,2 & 10,3 & 10,6 \\
\hline & $8,5 \%$ & 8,6 & 7,1 & 9,5 & 9,7 & 11,7 & 9,2 & 11,5 & 11,4 & 9,4 \\
\hline \multirow[t]{5}{*}{$-12^{\circ} \mathrm{C}$} & $21,1 \%$ & 22,9 & 23,9 & 21,5 & 24,0 & 24,6 & 22,3 & 22,1 & 17,2 & 22,3 \\
\hline & $15,9 \%$ & 15,4 & 18,8 & 16,7 & 18,2 & 18,4 & 16,5 & 15,7 & 16,2 & 16,5 \\
\hline & $13,6 \%$ & 12,6 & 10,1 & 13,8 & 13,8 & 14,2 & 12,4 & 12,6 & 12,3 & 12,7 \\
\hline & $11,9 \%$ & 9,9 & 9,7 & 9,1 & 8,8 & 8,6 & 10,1 & 9,1 & 9,20 & 9,8 \\
\hline & $8,5 \%$ & 7,5 & 7,8 & 8,1 & 8,9 & 9,5 & 8,0 & 8,5 & 7,80 & 8,1 \\
\hline
\end{tabular}

De modo similar aos resultados obtidos no teste de germinação, os testes de vigor (Tabelas 4, 5 e 6) permitiram identificar que os dois teores de água mais elevados na temperatura de armazenamento de $20^{\circ} \mathrm{C}$, conduziram a intenso processo de deterioração e, consequentemente, desfavorecendo a conservação das sementes. Dessa forma, as combinações de temperatura e teor de água de $11,9 \%$ a $10^{\circ} \mathrm{C}$; $11,9 \%$ a $-12^{\circ} \mathrm{C}$ e $13,6 \%$ a $-12{ }^{\circ} \mathrm{C}$ favoreceram a conservação das sementes. Oliveira et al. (2006) observaram, em sementes de ipê-amarelo-do-cerrado (Tabebuia aurea) armazenadas em embalagens de papel e em condições ambientais $( \pm$ $24^{\circ} \mathrm{C}$ ), que a viabilidade ultrapassou 90 dias. 
TABELA3. Germinação (\%) de sementes de Tabebuia chrysotricha (Mart. ex A. DC.) Standl. durante o armazenamento em diferentes condições de temperatura $\left(\mathrm{T},{ }^{\circ} \mathrm{C}\right)$ e de teor de água inicial das sementes $(\mathrm{TA}, \%)$.

\begin{tabular}{|c|c|c|c|c|c|c|c|c|c|c|}
\hline \multirow{2}{*}{$\begin{array}{l}\text { Fatores de } \\
\text { variação }\end{array}$} & \multirow{2}{*}{ Tratamentos } & \multicolumn{9}{|c|}{ Períodos de armazenamento (dias) } \\
\hline & & 30 & 60 & 90 & 120 & 150 & 180 & 210 & 240 & 280 \\
\hline \multirow[t]{5}{*}{ TA } & 21,1 & $7 \mathrm{c}$ & $2 \mathrm{~d}$ & $0 \mathrm{~d}$ & $0 \mathrm{~d}$ & $0 \mathrm{~d}$ & $0 \mathrm{e}$ & $0 \mathrm{~d}$ & $0 \mathrm{e}$ & $0 \mathrm{e}$ \\
\hline & 15,9 & $25 \mathrm{a}$ & $14 \mathrm{~b}$ & $2 \mathrm{c}$ & $1 \mathrm{c}$ & $1 \mathrm{c}$ & $1 \mathrm{~d}$ & $1 \mathrm{c}$ & $1 \mathrm{~d}$ & $1 \mathrm{~d}$ \\
\hline & 13,6 & $19 \mathrm{~b}$ & $17 \mathrm{~b}$ & $10 \mathrm{~b}$ & $6 \mathrm{~b}$ & $4 \mathrm{~b}$ & $4 \mathrm{c}$ & $4 \mathrm{~b}$ & $4 \mathrm{c}$ & $3 b$ \\
\hline & 11,9 & $28 \mathrm{a}$ & $30 \mathrm{a}$ & $28 \mathrm{a}$ & $34 \mathrm{a}$ & $20 \mathrm{a}$ & $22 \mathrm{a}$ & $17 \mathrm{a}$ & $18 \mathrm{a}$ & $15 \mathrm{a}$ \\
\hline & 8,5 & $10 \mathrm{c}$ & $6 c$ & $9 \mathrm{~b}$ & $5 \mathrm{~b}$ & $3 \mathrm{~b}$ & $8 \mathrm{~b}$ & $5 \mathrm{~b}$ & $5 \mathrm{~b}$ & $2 \mathrm{c}$ \\
\hline \multirow[t]{3}{*}{$\mathrm{T}$} & 10 & $17 \mathrm{a}$ & $13 \mathrm{~b}$ & $9 \mathrm{a}$ & $5 \mathrm{~b}$ & $1 \mathrm{c}$ & $6 \mathrm{~b}$ & $4 \mathrm{~b}$ & $4 \mathrm{~b}$ & $3 b$ \\
\hline & 20 & $13 \mathrm{~b}$ & $7 \mathrm{c}$ & $3 b$ & $2 c$ & $4 \mathrm{~b}$ & $1 \mathrm{c}$ & $1 \mathrm{c}$ & $1 \mathrm{c}$ & $1 \mathrm{c}$ \\
\hline & -12 & $21 \mathrm{a}$ & $17 \mathrm{a}$ & $9 \mathrm{a}$ & $10 \mathrm{a}$ & $8 \mathrm{a}$ & $8 \mathrm{a}$ & $10 \mathrm{a}$ & $9 \mathrm{a}$ & $7 \mathrm{a}$ \\
\hline \multirow[t]{16}{*}{$\mathrm{TA} \times \mathrm{T}$} & $21,1 / 10$ & $4 \mathrm{~d}$ & $0 \mathrm{~d}$ & $0 \mathrm{e}$ & $0 \mathrm{c}$ & $0 \mathrm{c}$ & $0 \mathrm{~d}$ & $0 \mathrm{~d}$ & $0 \mathrm{~d}$ & $0 \mathrm{~d}$ \\
\hline & $15,9 / 10$ & $29 a$ & $17 \mathrm{bc}$ & $4 \mathrm{~d}$ & $0 \mathrm{c}$ & $0 \mathrm{c}$ & $0 \mathrm{~d}$ & $0 \mathrm{~d}$ & $0 \mathrm{~d}$ & $0 \mathrm{~d}$ \\
\hline & $13,6 / 10$ & $19 \mathrm{bc}$ & $18 \mathrm{~b}$ & $11 \mathrm{c}$ & $6 \mathrm{~b}$ & $6 \mathrm{~b}$ & $4 \mathrm{c}$ & $4 c$ & $2 \mathrm{c}$ & $1 \mathrm{c}$ \\
\hline & $11,9 / 10$ & $28 \mathrm{ab}$ & $34 \mathrm{a}$ & $27 \mathrm{a}$ & $38 \mathrm{a}$ & $25 \mathrm{a}$ & $33 a$ & $21 \mathrm{a}$ & $29 a$ & $26 a$ \\
\hline & $8,5 / 10$ & $14 \mathrm{c}$ & $12 \mathrm{c}$ & $18 \mathrm{~b}$ & $7 \mathrm{~b}$ & $5 \mathrm{~b}$ & $16 \mathrm{~b}$ & $13 \mathrm{~b}$ & $10 \mathrm{~b}$ & $5 \mathrm{~b}$ \\
\hline & $21,1 / 20$ & $1 \mathrm{c}$ & $0 \mathrm{~d}$ & $0 \mathrm{~d}$ & $0 \mathrm{c}$ & $0 \mathrm{c}$ & $0 \mathrm{c}$ & $0 \mathrm{~b}$ & $1 \mathrm{~b}$ & $0 \mathrm{~b}$ \\
\hline & $15,9 / 20$ & $17 \mathrm{~b}$ & $3 c$ & $0 \mathrm{~d}$ & $0 \mathrm{c}$ & $0 \mathrm{c}$ & $0 \mathrm{c}$ & $0 \mathrm{~b}$ & $0 \mathrm{c}$ & $0 \mathrm{~b}$ \\
\hline & $13,6 / 20$ & $12 \mathrm{~b}$ & $10 \mathrm{~b}$ & $2 c$ & $0 \mathrm{c}$ & $0 \mathrm{c}$ & $0 \mathrm{c}$ & $0 \mathrm{~b}$ & $0 \mathrm{c}$ & $0 \mathrm{~b}$ \\
\hline & $11,9 / 20$ & $34 \mathrm{a}$ & $27 \mathrm{a}$ & $23 \mathrm{a}$ & $25 \mathrm{a}$ & $14 \mathrm{a}$ & $14 \mathrm{a}$ & $7 \mathrm{a}$ & $3 \mathrm{a}$ & $2 \mathrm{a}$ \\
\hline & $8,5 / 20$ & $12 \mathrm{~b}$ & $7 \mathrm{bc}$ & $7 \mathrm{~b}$ & $5 \mathrm{~b}$ & $1 \mathrm{~b}$ & $4 \mathrm{~b}$ & $0 \mathrm{~b}$ & $1 \mathrm{~b}$ & $0 \mathrm{~b}$ \\
\hline & $21,1 /-12$ & $20 \mathrm{~b}$ & $15 \mathrm{~b}$ & $0 \mathrm{e}$ & $0 \mathrm{e}$ & $0 \mathrm{~d}$ & $0 \mathrm{~d}$ & $0 \mathrm{~d}$ & $0 \mathrm{~d}$ & $0 \mathrm{~d}$ \\
\hline & $15,9 /-12$ & $31 \mathrm{a}$ & $27 \mathrm{a}$ & $8 \mathrm{c}$ & $8 \mathrm{c}$ & $10 \mathrm{bc}$ & $7 c$ & $5 \mathrm{c}$ & $7 c$ & $2 d$ \\
\hline & $13,6 /-12$ & $29 a b$ & $24 \mathrm{a}$ & $20 \mathrm{~b}$ & $20 \mathrm{~b}$ & $13 b$ & $16 \mathrm{~b}$ & $20 \mathrm{a}$ & $19 \mathrm{~b}$ & $18 \mathrm{~b}$ \\
\hline & $11,9 /-12$ & $22 \mathrm{ab}$ & $30 \mathrm{a}$ & $33 \mathrm{a}$ & $39 \mathrm{a}$ & $21 \mathrm{a}$ & $21 \mathrm{a}$ & $24 \mathrm{a}$ & $28 \mathrm{a}$ & $26 \mathrm{a}$ \\
\hline & $8,5 /-12$ & $6 \mathrm{c}$ & $1 \mathrm{c}$ & $4 \mathrm{~d}$ & $4 \mathrm{~d}$ & $7 \mathrm{c}$ & $6 \mathrm{c}$ & $9 \mathrm{~b}$ & $8 \mathrm{c}$ & $5 \mathrm{c}$ \\
\hline & $\mathrm{CV}(\%)$ & 19,2 & 18,3 & 18,5 & 18,0 & 21,8 & 20,8 & 26,0 & 22,8 & 21,7 \\
\hline
\end{tabular}

Médias seguidas de mesma letra na coluna não diferem entre si pelo teste de Tukey a $5 \%$ de probabilidade. CV - coeficiente de varação experimental 
TABELA 4. Emergência de plântulas (\%) de Tabebuia chrysotricha (Mart. ex A. DC.) Standl. durante o armazenamento em diferentes condições de temperatura $\left(T,{ }^{\circ} \mathrm{C}\right)$ e de teor de água inicial das sementes (TA, \%).

\begin{tabular}{|c|c|c|c|c|c|c|c|c|c|c|}
\hline \multirow{2}{*}{$\begin{array}{c}\text { Fatores de } \\
\text { variação }\end{array}$} & \multirow[b]{2}{*}{ Tratamentos } & \multicolumn{9}{|c|}{ Períodos de armazenamento (dias) } \\
\hline & & 30 & 60 & 90 & 120 & 150 & 180 & 210 & 240 & 280 \\
\hline \multirow[t]{5}{*}{ TA } & 21,1 & $5 \mathrm{c}$ & $1 \mathrm{c}$ & $0 \mathrm{~d}$ & $0 \mathrm{~d}$ & $0 \mathrm{~d}$ & $0 \mathrm{~d}$ & $0 \mathrm{e}$ & $0 \mathrm{~d}$ & $0 \mathrm{~d}$ \\
\hline & 15,9 & $21 \mathrm{a}$ & $9 \mathrm{~b}$ & $5 \mathrm{c}$ & $2 \mathrm{c}$ & $1 \mathrm{c}$ & $1 \mathrm{c}$ & $1 \mathrm{~d}$ & $0 \mathrm{~d}$ & $1 \mathrm{c}$ \\
\hline & 13,6 & $12 \mathrm{~b}$ & $10 \mathrm{~b}$ & $10 \mathrm{~b}$ & $9 \mathrm{~b}$ & $4 \mathrm{~b}$ & $2 b$ & $2 \mathrm{c}$ & $1 \mathrm{c}$ & $1 \mathrm{c}$ \\
\hline & 11,9 & $19 \mathrm{a}$ & $22 \mathrm{a}$ & $23 \mathrm{a}$ & $22 \mathrm{a}$ & $18 \mathrm{a}$ & $10 \mathrm{a}$ & $7 \mathrm{a}$ & $6 \mathrm{a}$ & $5 \mathrm{a}$ \\
\hline & 8,5 & $7 \mathrm{c}$ & $9 \mathrm{~b}$ & $10 \mathrm{~b}$ & $7 \mathrm{~b}$ & $5 \mathrm{~b}$ & $4 \mathrm{~b}$ & $4 \mathrm{~b}$ & $2 \mathrm{~b}$ & $4 \mathrm{~b}$ \\
\hline \multirow[t]{3}{*}{$\mathrm{T}$} & 10 & $9 \mathrm{~b}$ & $6 \mathrm{~b}$ & $11 \mathrm{a}$ & $6 \mathrm{~b}$ & $3 \mathrm{~b}$ & $1 \mathrm{~b}$ & $2 b$ & $0 \mathrm{c}$ & $2 b$ \\
\hline & 20 & $10 \mathrm{~b}$ & $8 \mathrm{ab}$ & $4 c$ & $2 \mathrm{c}$ & $2 \mathrm{c}$ & $0 \mathrm{c}$ & $0 \mathrm{c}$ & $1 \mathrm{~b}$ & $0 \mathrm{c}$ \\
\hline & -12 & $16 \mathrm{a}$ & $10 \mathrm{a}$ & $8 \mathrm{~b}$ & $11 \mathrm{a}$ & $6 \mathrm{a}$ & $9 \mathrm{a}$ & $5 \mathrm{a}$ & $3 \mathrm{a}$ & $8 \mathrm{a}$ \\
\hline \multirow[t]{16}{*}{$\mathrm{TA} \times \mathrm{T}$} & $21,1 / 10$ & $1 \mathrm{c}$ & $0 \mathrm{c}$ & $0 \mathrm{~d}$ & $0 \mathrm{c}$ & $0 \mathrm{~d}$ & $0 \mathrm{c}$ & $0 \mathrm{~b}$ & $0 \mathrm{c}$ & $0 \mathrm{c}$ \\
\hline & $15,9 / 10$ & $19 \mathrm{a}$ & $11 \mathrm{~b}$ & $9 \mathrm{c}$ & $0 \mathrm{c}$ & $0 \mathrm{~d}$ & $0 \mathrm{c}$ & $0 \mathrm{~b}$ & $0 \mathrm{c}$ & $0 \mathrm{c}$ \\
\hline & $13,6 / 10$ & $8 \mathrm{~b}$ & $8 \mathrm{~b}$ & $16 \mathrm{~b}$ & $25 \mathrm{a}$ & $3 c$ & $0 \mathrm{c}$ & $0 \mathrm{~b}$ & $0 \mathrm{c}$ & $0 \mathrm{c}$ \\
\hline & $11,9 / 10$ & $14 \mathrm{ab}$ & $23 a$ & $24 \mathrm{a}$ & $15 \mathrm{~b}$ & $23 \mathrm{a}$ & $12 \mathrm{a}$ & $10 \mathrm{a}$ & $11 \mathrm{a}$ & $21 \mathrm{a}$ \\
\hline & $8,5 / 10$ & $8 \mathrm{~b}$ & $11 \mathrm{~b}$ & $20 \mathrm{ab}$ & $12 \mathrm{~b}$ & $6 \mathrm{~b}$ & $6 \mathrm{~b}$ & $9 \mathrm{a}$ & $5 \mathrm{~b}$ & $9 \mathrm{~b}$ \\
\hline & $21,1 / 20$ & $1 \mathrm{c}$ & $0 \mathrm{~d}$ & $0 \mathrm{~d}$ & $0 \mathrm{c}$ & $0 \mathrm{~b}$ & $0 \mathrm{~b}$ & $0 \mathrm{c}$ & $0 \mathrm{~b}$ & $0 \mathrm{a}$ \\
\hline & $15,9 / 20$ & $14 a b$ & $1 \mathrm{c}$ & $0 \mathrm{~d}$ & $0 \mathrm{c}$ & $0 \mathrm{~b}$ & $0 \mathrm{~b}$ & $0 \mathrm{c}$ & $0 \mathrm{~b}$ & $0 \mathrm{a}$ \\
\hline & $13,6 / 20$ & $14 \mathrm{ab}$ & $6 \mathrm{~b}$ & $1 \mathrm{c}$ & $0 \mathrm{c}$ & $0 \mathrm{~b}$ & $0 \mathrm{~b}$ & $0 \mathrm{c}$ & $0 \mathrm{~b}$ & $0 \mathrm{a}$ \\
\hline & $11,9 / 20$ & $22 \mathrm{a}$ & $23 \mathrm{a}$ & $27 \mathrm{a}$ & $20 \mathrm{a}$ & $13 \mathrm{a}$ & $2 \mathrm{a}$ & $2 \mathrm{a}$ & $1 \mathrm{a}$ & $0 \mathrm{a}$ \\
\hline & $8,5 / 20$ & $7 \mathrm{~b}$ & $18 \mathrm{a}$ & $11 \mathrm{~b}$ & $7 \mathrm{~b}$ & $9 \mathrm{a}$ & $2 \mathrm{a}$ & $1 \mathrm{~b}$ & $0 \mathrm{~b}$ & $0 \mathrm{a}$ \\
\hline & $21,1 /-12$ & $18 \mathrm{~b}$ & $3 \mathrm{~b}$ & $0 \mathrm{c}$ & $1 \mathrm{~d}$ & $0 \mathrm{~d}$ & $0 \mathrm{c}$ & $0 \mathrm{c}$ & $0 \mathrm{~d}$ & $0 \mathrm{c}$ \\
\hline & $15,9 /-12$ & $30 \mathrm{a}$ & $17 \mathrm{a}$ & $16 \mathrm{a}$ & $17 \mathrm{~b}$ & $5 \mathrm{~b}$ & $9 \mathrm{~b}$ & $5 \mathrm{~b}$ & $3 c$ & $9 \mathrm{~b}$ \\
\hline & $13,6 /-12$ & $13 \mathrm{bc}$ & $18 \mathrm{a}$ & $19 \mathrm{a}$ & $15 \mathrm{~b}$ & $17 \mathrm{a}$ & $22 \mathrm{a}$ & $10 \mathrm{a}$ & $7 \mathrm{~b}$ & $12 \mathrm{~b}$ \\
\hline & $11,9 /-12$ & $19 a b$ & $21 \mathrm{a}$ & $19 \mathrm{a}$ & $32 \mathrm{a}$ & $19 \mathrm{a}$ & $24 \mathrm{a}$ & $12 \mathrm{a}$ & $10 \mathrm{a}$ & $20 a$ \\
\hline & $8,5 /-12$ & $6 \mathrm{c}$ & $2 b$ & $3 \mathrm{~b}$ & $4 \mathrm{c}$ & $1 \mathrm{c}$ & $6 \mathrm{~b}$ & $5 \mathrm{~b}$ & $3 c$ & $10 \mathrm{~b}$ \\
\hline & $\mathrm{CV}(\%)$ & 21,6 & 19,7 & 16,3 & 15,9 & 19,8 & 23,2 & 14,5 & 18,1 & 23,5 \\
\hline
\end{tabular}

Médias seguidas de mesma letra na coluna não diferem entre si pelo teste de Tukey a 5\% de probabilidade. CV - coeficiente de varação experimental 
TABELA 5. Índice de velocidade de emergência de plântulas de Tabebuia chrysotricha (Mart. ex A. DC.) Standl. (ipêamarelo) durante o armazenamento em diferentes condições de temperatura $\left(T,{ }^{\circ} \mathrm{C}\right)$ e de teor de água inicial das sementes (TA, \%).

\begin{tabular}{|c|c|c|c|c|c|c|c|c|c|c|}
\hline \multirow{2}{*}{$\begin{array}{c}\text { Fatores de } \\
\text { variação }\end{array}$} & \multirow[b]{2}{*}{ Tratamentos } & \multicolumn{9}{|c|}{ Períodos de armazenamento (dias) } \\
\hline & & 30 & 60 & 90 & 120 & 150 & 180 & 210 & 240 & 280 \\
\hline \multirow[t]{5}{*}{ TA } & 21,1 & $0,13 \mathrm{c}$ & $0,15 \mathrm{c}$ & $0,00 \mathrm{~d}$ & $0,00 \mathrm{c}$ & $0,00 \mathrm{c}$ & $0,00 \mathrm{~d}$ & $0,00 \mathrm{~d}$ & $0,00 \mathrm{c}$ & $0,00 \mathrm{c}$ \\
\hline & 15,9 & $0,29 a b$ & $0,23 \mathrm{bc}$ & $0,15 \mathrm{c}$ & $0,00 \mathrm{c}$ & $0,02 \mathrm{c}$ & $0,06 \mathrm{c}$ & $0,01 \mathrm{~cd}$ & $0,01 \mathrm{bc}$ & $0,06 \mathrm{bc}$ \\
\hline & 13,6 & $0,21 \mathrm{bc}$ & $0,24 \mathrm{bc}$ & $0,21 \mathrm{~b}$ & $0,53 \mathrm{a}$ & $0,09 \mathrm{~b}$ & $0,11 \mathrm{~b}$ & $0,06 \mathrm{~b}$ & $0,02 \mathrm{~b}$ & $0,11 \mathrm{bc}$ \\
\hline & 11,9 & $0,33 \mathrm{a}$ & $0,52 \mathrm{a}$ & $0,43 \mathrm{a}$ & $0,21 \mathrm{~b}$ & $0,17 \mathrm{a}$ & $0,23 \mathrm{a}$ & $0,17 \mathrm{a}$ & $0,07 \mathrm{a}$ & $0,59 \mathrm{a}$ \\
\hline & 8,5 & $0,15 \mathrm{c}$ & $0,34 \mathrm{~b}$ & $0,20 \mathrm{~b}$ & $0,19 \mathrm{~b}$ & $0,09 \mathrm{~b}$ & $0,09 \mathrm{bc}$ & $0,03 \mathrm{bc}$ & $0,01 \mathrm{bc}$ & $0,19 \mathrm{~b}$ \\
\hline \multirow[t]{3}{*}{$\mathrm{T}$} & 10 & $0,16 \mathrm{~b}$ & $0,22 \mathrm{~b}$ & $0,23 \mathrm{a}$ & $0,18 \mathrm{~b}$ & $0,06 \mathrm{~b}$ & $0,07 \mathrm{~b}$ & $0,10 \mathrm{a}$ & $0,02 \mathrm{~b}$ & $0,24 \mathrm{a}$ \\
\hline & 20 & $0,18 \mathrm{~b}$ & $0,28 a b$ & $0,17 \mathrm{c}$ & $0,09 \mathrm{c}$ & $0,05 \mathrm{~b}$ & $0,02 \mathrm{c}$ & $0,01 \mathrm{c}$ & $0,00 \mathrm{c}$ & $0,00 \mathrm{~b}$ \\
\hline & -12 & $0,32 \mathrm{a}$ & $0,38 \mathrm{a}$ & $0,20 \mathrm{~b}$ & $0,29 \mathrm{a}$ & $0,11 \mathrm{a}$ & $0,20 \mathrm{a}$ & $0,05 \mathrm{~b}$ & $0,04 \mathrm{a}$ & $0,33 \mathrm{a}$ \\
\hline \multirow[t]{16}{*}{$\mathrm{TA} \times \mathrm{T}$} & $21,1 / 10$ & $0,07 a b$ & $0,00 \mathrm{~b}$ & $0,00 \mathrm{~d}$ & $0,00 \mathrm{c}$ & $0,00 \mathrm{c}$ & $0,00 \mathrm{c}$ & $0,00 \mathrm{c}$ & $0,00 \mathrm{~b}$ & $0,00 \mathrm{~b}$ \\
\hline & $15,9 / 10$ & $0,21 a b$ & $0,26 a b$ & $0,19 \mathrm{c}$ & $0,00 \mathrm{c}$ & $0,00 \mathrm{c}$ & $0,00 \mathrm{c}$ & $0,00 \mathrm{c}$ & $0,00 \mathrm{~b}$ & $0,00 \mathrm{~b}$ \\
\hline & $13,6 / 10$ & $0,11 \mathrm{ab}$ & $0,13 \mathrm{~b}$ & $0,27 \mathrm{~b}$ & $0,53 \mathrm{a}$ & $0,07 \mathrm{~b}$ & $0,00 \mathrm{c}$ & $0,10 \mathrm{~b}$ & $0,00 \mathrm{~b}$ & $0,00 \mathrm{~b}$ \\
\hline & $11,9 / 10$ & $0,27 \mathrm{a}$ & $0,48 \mathrm{a}$ & $0,40 \mathrm{a}$ & $0,21 \mathrm{~b}$ & $0,17 \mathrm{a}$ & $0,23 \mathrm{a}$ & $0,36 \mathrm{a}$ & $0,11 \mathrm{a}$ & $1,05 \mathrm{a}$ \\
\hline & $8,5 / 10$ & $0,15 a b$ & $0,25 \mathrm{ab}$ & $0,31 \mathrm{~b}$ & $0,19 \mathrm{~b}$ & $0,09 \mathrm{~b}$ & $0,12 \mathrm{~b}$ & $0,07 \mathrm{~b}$ & $0,02 \mathrm{~b}$ & $0,15 \mathrm{~b}$ \\
\hline & $21,1 / 20$ & $0,07 \mathrm{~b}$ & $0,00 \mathrm{~b}$ & $0,00 \mathrm{~d}$ & $0,00 \mathrm{c}$ & $0,00 \mathrm{~b}$ & $0,00 \mathrm{~b}$ & $0,00 \mathrm{~b}$ & $0,00 \mathrm{a}$ & $0,00 \mathrm{a}$ \\
\hline & $15,9 / 20$ & $0,14 \mathrm{~b}$ & $0,11 \mathrm{~b}$ & $0,00 \mathrm{~d}$ & $0,00 \mathrm{c}$ & $0,00 \mathrm{~b}$ & $0,00 \mathrm{~b}$ & $0,00 \mathrm{~b}$ & $0,00 \mathrm{a}$ & $0,00 \mathrm{a}$ \\
\hline & $13,6 / 20$ & $0,21 \mathrm{ab}$ & $0,13 \mathrm{~b}$ & $0,07 \mathrm{c}$ & $0,00 \mathrm{c}$ & $0,00 \mathrm{~b}$ & $0,00 \mathrm{~b}$ & $0,00 \mathrm{~b}$ & $0,00 \mathrm{a}$ & $0,00 \mathrm{a}$ \\
\hline & $11,9 / 20$ & $0,36 \mathrm{a}$ & $0,64 \mathrm{a}$ & $0,59 \mathrm{a}$ & $0,30 \mathrm{a}$ & $0,15 \mathrm{a}$ & $0,10 \mathrm{a}$ & $0,05 \mathrm{a}$ & $0,01 \mathrm{a}$ & $0,00 \mathrm{a}$ \\
\hline & 8,5 / 20 & $0,10 \mathrm{~b}$ & $0,51 \mathrm{a}$ & $0,19 \mathrm{~b}$ & $0,15 \mathrm{~b}$ & $0,10 \mathrm{a}$ & $0,00 \mathrm{~b}$ & $0,00 \mathrm{~b}$ & $0,00 \mathrm{a}$ & $0,00 \mathrm{a}$ \\
\hline & $21,1 /-12$ & $0,24 \mathrm{~b}$ & $0,44 \mathrm{a}$ & $0,00 \mathrm{c}$ & $0,00 \mathrm{e}$ & $0,00 \mathrm{c}$ & $0,00 \mathrm{c}$ & $0,00 \mathrm{~b}$ & $0,00 \mathrm{c}$ & $0,00 \mathrm{c}$ \\
\hline & $15,9 /-12$ & $0,52^{\mathrm{a}}$ & $0,31 \mathrm{a}$ & $0,27 \mathrm{a}$ & $0,25 \mathrm{c}$ & $0,06 \mathrm{~b}$ & $0,19 \mathrm{~b}$ & $0,05 \mathrm{a}$ & $0,04 \mathrm{~b}$ & $0,18 b c$ \\
\hline & $13,6 /-12$ & $0,31 \mathrm{~b}$ & $0,48 \mathrm{a}$ & $0,31 \mathrm{a}$ & $0,43 \mathrm{~b}$ & $0,20 \mathrm{a}$ & $0,35 \mathrm{a}$ & $0,09 \mathrm{a}$ & $0,07 \mathrm{a}$ & $0,33 \mathrm{~b}$ \\
\hline & $11,9 /-12$ & $0,36 a b$ & $0,45 \mathrm{a}$ & $0,31 \mathrm{a}$ & $0,67 \mathrm{a}$ & $0,19 \mathrm{a}$ & $0,37 \mathrm{a}$ & $0,07 \mathrm{a}$ & $0,09 \mathrm{a}$ & $0,72 \mathrm{a}$ \\
\hline & $8,5 /-12$ & $0,20 \mathrm{~b}$ & $0,24 \mathrm{a}$ & $0,11 \mathrm{~b}$ & $0,11 \mathrm{~d}$ & $0,09 \mathrm{~b}$ & $0,13 \mathrm{~b}$ & $0,04 \mathrm{ab}$ & $0,03 \mathrm{bc}$ & $0,44 a b$ \\
\hline & $\mathrm{CV}(\%)$ & 27,0 & 25,2 & 18,3 & 24,5 & 19,1 & 29,0 & 26,5 & 29,3 & 29,8 \\
\hline
\end{tabular}

Médias seguidas de mesma letra na coluna não diferem entre si pelo teste de Tukey a 5\% de probabilidade. CV - coeficiente de varação experimental 
TABELA 6. Comprimento da parte aérea de plântulas (cm) de Tabebuia chrysotricha (Mart. ex A. DC.) Standl. durante o armazenamento em diferentes condições de temperatura $\left(\mathrm{T},{ }^{\circ} \mathrm{C}\right)$ e de teor de água inicial das sementes (TA, \%).

\begin{tabular}{|c|c|c|c|c|c|c|c|c|c|c|}
\hline \multirow{2}{*}{$\begin{array}{c}\text { Fatores de } \\
\text { variação }\end{array}$} & \multirow{2}{*}{ Tratamentos } & \multicolumn{9}{|c|}{ Períodos de armazenamento (dias) } \\
\hline & & 30 & 60 & 90 & 120 & 150 & 180 & 210 & 240 & 280 \\
\hline \multirow[t]{5}{*}{ TA } & 21,1 & $0,29 \mathrm{~b}$ & $0,02 \mathrm{~d}$ & $0,00 \mathrm{~d}$ & $0,03 \mathrm{~d}$ & $0,00 \mathrm{c}$ & $0,00 \mathrm{c}$ & $0,00 \mathrm{c}$ & $0,00 \mathrm{c}$ & $0,00 \mathrm{c}$ \\
\hline & 15,9 & $0,63 \mathrm{a}$ & $0,49 \mathrm{~b}$ & $0,24 \mathrm{c}$ & $0,18 \mathrm{~cd}$ & $0,05 \mathrm{c}$ & $0,12 b$ & $0,03 \mathrm{c}$ & $0,04 \mathrm{~b}$ & $0,12 b$ \\
\hline & 13,6 & $0,43 \mathrm{ab}$ & $0,35 \mathrm{c}$ & $0,28 \mathrm{c}$ & $0,35 \mathrm{~b}$ & $0,14 \mathrm{~b}$ & $0,16 \mathrm{~b}$ & $0,05 \mathrm{c}$ & $0,05 \mathrm{~b}$ & $0,14 \mathrm{~b}$ \\
\hline & 11,9 & $0,61 \mathrm{a}$ & $0,64 \mathrm{a}$ & $0,79 \mathrm{a}$ & $0,70 \mathrm{a}$ & $0,35 \mathrm{a}$ & $0,33 \mathrm{a}$ & $0,32 \mathrm{a}$ & $0,13 \mathrm{a}$ & $0,37 \mathrm{a}$ \\
\hline & 8,5 & $0,22 \mathrm{~b}$ & $0,58 \mathrm{ab}$ & $0,35 \mathrm{~b}$ & $0,32 \mathrm{bc}$ & $0,12 \mathrm{~b}$ & $0,11 \mathrm{~b}$ & $0,13 \mathrm{~b}$ & $0,04 \mathrm{~b}$ & $0,19 \mathrm{~b}$ \\
\hline \multirow[t]{3}{*}{$\mathrm{T}$} & 10 & $0,30 \mathrm{~b}$ & $0,29 \mathrm{c}$ & $0,35 \mathrm{a}$ & $0,25 \mathrm{~b}$ & $0,13 \mathrm{~b}$ & $0,11 \mathrm{~b}$ & $0,18 \mathrm{a}$ & $0,04 \mathrm{~b}$ & $0,20 \mathrm{~b}$ \\
\hline & 20 & $0,36 \mathrm{~b}$ & $0,57 \mathrm{a}$ & $0,30 \mathrm{~b}$ & $0,15 \mathrm{c}$ & $0,07 \mathrm{c}$ & $0,01 \mathrm{c}$ & $0,03 \mathrm{c}$ & $0,01 \mathrm{c}$ & $0,00 \mathrm{c}$ \\
\hline & -12 & $0,64 \mathrm{a}$ & $0,38 \mathrm{~b}$ & $0,35 \mathrm{a}$ & $0,54 \mathrm{a}$ & $0,19 \mathrm{a}$ & $0,31 \mathrm{a}$ & $0,11 \mathrm{~b}$ & $0,11 \mathrm{a}$ & $0,29 \mathrm{a}$ \\
\hline \multirow[t]{16}{*}{$\mathrm{TA} \times \mathrm{T}$} & $21,1 / 10$ & $0,08 \mathrm{a}$ & $0,00 \mathrm{~b}$ & $0,00 \mathrm{~d}$ & $0,00 \mathrm{~b}$ & $0,00 \mathrm{c}$ & $0,00 \mathrm{c}$ & $0,00 \mathrm{c}$ & $0,00 \mathrm{c}$ & $0,00 \mathrm{c}$ \\
\hline & $15,9 / 10$ & $0,38 \mathrm{a}$ & $0,30 \mathrm{a}$ & $0,18 \mathrm{c}$ & $0,00 \mathrm{~b}$ & $0,00 \mathrm{c}$ & $0,00 \mathrm{c}$ & $0,00 \mathrm{c}$ & $0,00 \mathrm{c}$ & $0,00 \mathrm{c}$ \\
\hline & $13,6 / 10$ & $0,44 \mathrm{a}$ & $0,28 \mathrm{a}$ & $0,41 \mathrm{~b}$ & $0,50 \mathrm{a}$ & $0,00 \mathrm{c}$ & $0,00 \mathrm{c}$ & $0,00 \mathrm{c}$ & $0,00 \mathrm{c}$ & $0,00 \mathrm{c}$ \\
\hline & $11,9 / 10$ & $0,43 \mathrm{a}$ & $0,46 \mathrm{a}$ & $0,59 \mathrm{a}$ & $0,42 \mathrm{a}$ & $0,51 \mathrm{a}$ & $0,39 \mathrm{a}$ & $0,60 \mathrm{a}$ & $0,20 \mathrm{a}$ & $0,68 \mathrm{a}$ \\
\hline & $8,5 / 10$ & $0,18 \mathrm{a}$ & $0,44 \mathrm{a}$ & $0,56 \mathrm{a}$ & $0,36 \mathrm{a}$ & $0,16 \mathrm{~b}$ & $0,16 \mathrm{~b}$ & $0,29 \mathrm{~b}$ & $0,03 \mathrm{~b}$ & $0,34 \mathrm{~b}$ \\
\hline & $21,1 / 20$ & $0,10 \mathrm{~b}$ & $0,00 \mathrm{~d}$ & $0,00 \mathrm{~d}$ & $0,00 \mathrm{~b}$ & $0,00 \mathrm{~b}$ & $0,00 \mathrm{~b}$ & $0,00 \mathrm{c}$ & $0,00 \mathrm{~b}$ & $0,00 \mathrm{a}$ \\
\hline & $15,9 / 20$ & $0,31 \mathrm{~b}$ & $0,64 \mathrm{~b}$ & $0,00 \mathrm{~d}$ & $0,00 \mathrm{~b}$ & $0,00 \mathrm{~b}$ & $0,00 \mathrm{~b}$ & $0,00 \mathrm{c}$ & $0,00 \mathrm{~b}$ & $0,00 \mathrm{a}$ \\
\hline & $13,6 / 20$ & $0,35 a b$ & $0,24 \mathrm{c}$ & $0,03 \mathrm{c}$ & $0,00 \mathrm{~b}$ & $0,00 \mathrm{~b}$ & $0,00 \mathrm{~b}$ & $0,00 \mathrm{c}$ & $0,00 \mathrm{~b}$ & $0,00 \mathrm{a}$ \\
\hline & $11,9 / 20$ & $0,79 \mathrm{a}$ & $0,82 \mathrm{~b}$ & $1,07 \mathrm{a}$ & $0,48 \mathrm{a}$ & $0,22 \mathrm{a}$ & $0,04 \mathrm{a}$ & $0,16 \mathrm{a}$ & $0,05 \mathrm{a}$ & $0,00 \mathrm{a}$ \\
\hline & $8,5 / 20$ & $0,26 \mathrm{~b}$ & $1,18 \mathrm{a}$ & $0,40 \mathrm{~b}$ & $0,29 \mathrm{a}$ & $0,15 \mathrm{a}$ & $0,02 \mathrm{a}$ & $0,01 \mathrm{~b}$ & $0,00 \mathrm{~b}$ & $0,00 \mathrm{a}$ \\
\hline & $21,1 /-12$ & $0,68 \mathrm{~b}$ & $0,08 \mathrm{~b}$ & $0,00 \mathrm{e}$ & $0,10 \mathrm{~d}$ & $0,00 \mathrm{c}$ & $0,00 \mathrm{~d}$ & $0,00 \mathrm{~b}$ & $0,00 \mathrm{~b}$ & $0,00 \mathrm{c}$ \\
\hline & $15,9 /-12$ & $1,20 \mathrm{a}$ & $0,54 \mathrm{a}$ & $0,54 \mathrm{~b}$ & $0,53 \mathrm{bc}$ & $0,15 \mathrm{~b}$ & $0,36 \mathrm{~b}$ & $0,10 a b$ & $0,14 \mathrm{a}$ & $0,39 \mathrm{ab}$ \\
\hline & $13,6 /-12$ & $0,49 \mathrm{~b}$ & $0,54 \mathrm{a}$ & $0,41 \mathrm{c}$ & $0,57 \mathrm{~b}$ & $0,42 \mathrm{a}$ & $0,49 a b$ & $0,15 \mathrm{a}$ & $0,17 \mathrm{a}$ & $0,44 \mathrm{a}$ \\
\hline & $11,9 /-12$ & $0,63 \mathrm{~b}$ & $0,63 \mathrm{a}$ & $0,72 \mathrm{a}$ & $1,20 \mathrm{a}$ & $0,32 \mathrm{a}$ & $0,57 \mathrm{a}$ & $0,20 \mathrm{a}$ & $0,14 \mathrm{a}$ & $0,43 \mathrm{a}$ \\
\hline & $8,5 /-12$ & $0,21 \mathrm{~b}$ & $0,13 \mathrm{~b}$ & $0,08 \mathrm{~d}$ & $0,30 \mathrm{~cd}$ & $0,05 \mathrm{bc}$ & $0,17 \mathrm{c}$ & $0,10 a b$ & $0,11 \mathrm{a}$ & $0,22 \mathrm{~b}$ \\
\hline & $\mathrm{CV}(\%)$ & 29,8 & 30,0 & 15,8 & 26,5 & 26,8 & 31,2 & 29,7 & 27,4 & 24,7 \\
\hline
\end{tabular}

Médias seguidas de mesma letra na coluna não diferem entre si pelo teste de Tukey a 5\% de probabilidade. CV - coeficiente de varação experimental

\section{CONCLUSÕES}

A conservação das sementes de ipê-amarelo (Tabebuia chrysotricha) é favorecida quando estas são armazenadas com teor de água em torno de $11,9 \%$ nas temperaturas de $10^{\circ} \mathrm{C} \mathrm{e}-12^{\circ} \mathrm{C}$.

\section{REFERÊNCIAS}

AGUIAR, I.B.; PIÑA-RODRIGUES, F.C.M.; FIGLIOLIA, M.B. Sementes florestais tropicais. Brasília: Abrates, 1993. $350 \mathrm{p}$.

BRASIL, Ministério da Agricultura e Reforma Agrária. 
Regras para análise de sementes. Brasília: SNDA/DNDV/ CLAV, 1992. 365p.

CARVALHO, N.M.; GÓES, M.; AGUIAR, I.B.; FERNANDES, P.D. Armazenamento de sementes de ipêamarelo (Tabebuia chrysotricha). Científica, v.4, n.3, p.315319, 1976.

CARVALHO, P.E.R. Espécies florestais brasileiras: recomendações silviculturais, potencialidades e uso da madeira. Brasília: EMBRAPA-CNPF, 1994. p.8-15.

CROMARTY, A.S.; ELLIS, R.H.; ROBERTS, E.H. Design of seed storage facilities for genetic conservation. Rome: International Board of Plant Genetic Resources, 1985. 100p.

DEGAN, P.; AGUIAR, I.B.; SADER, R.; PERECIN, D.; PINTO, L.R. Influência de métodos de secagem na conservação de sementes de ipê-branco. Revista Brasileira de Engenharia Agrícola e Ambiental, v.5, n.3, p.492-496, 2001.

FIGLIOLIA, M.B.; SILVA, A.; JARDIM, D.C.P.; IWANE, M.S.S. Viabilidade de sementes liofilizadas de essências florestais nativas. Silvicultura em São Paulo, v. 20/22, p. 47-55, 1986/88.

KAGEYAMA, P.Y.; MARQUES, F.C.M. Comportamento de sementes de curta longevidade armazenadas com diferentes teores de umidade inicial: gênero Tabebuia. México: Instituto Nacional de Investigaciones Forestale, 1981. p.347-352 (Publicación Especial, n.35)

KANO, N.K.; MARQUES, F.C.M.; KAGEYAMA, P.Y. Armazenamento de sementes de ipê-dourado (Tabebuia sp.). Revista: IPEF, v.1, n.17, p.12-33, 1978.

LORENZI, H. Árvores brasileiras: manual de identificação e cultivo de plantas arbóreas nativas do Brasil. Nova Odessa:
Editora Plantarum, 2002. v.1, p.64.

MARCOS FILHO, J.; CÍCERO, S.M.; SILVA, W.R. Avaliação da qualidade das sementes. Piracicaba: FEALQ, 1987.230p.

MELLO, C.M.C.; EIRA, M.T.S. Conservação de sementes de ipês (Tabebuia spp.). Revista Árvore,v.19, n.4, p.427432, 1995.

NATALE, W.; CARVALHO, N.M. A liofilização como método de secagem de sementes de ipê-roxo (Tabebuia sp.).

Revista Brasileira de Armazenamento, v.8, n.1/2, p.35-37, 1983.

OLIVEIRA, A.K.M.; SCHLEDER, E.D.; FAVERO, S. Caracterização morfológica, viabilidade e vigor de sementes de Tabebuia aurea (Silva Manso) Benth. \& Hook. F. ex. S. Moore. Revista Árvore, v.30, n.1, p.25-32, 2006.

PINTO, M.M.; SADER, R.; BARBOSA, J.M. Influência do tempo de secagem e do armazenamento sobre a viabilidade das sementes de ipê-rosa. Revista Brasileira de Sementes, v. 8, n. 1, p. $37-47,1986$.

ROBERTS, E.H. Predicting the storage life of seeds. Seed Science and Technology, v. 1, n.1, p.499-514, 1973.

SOCOLOWSKI, F.; TAKAKI, M. Germinação de sementes e emergência de plântulas de Tabebuia rosea (Bertolini) A.P. de Candolle (Bignoniaceae), uma espécie exótica com potencial invasor. Revista Árvore, v.31, n.2, p.229-238, 2007.

VANZOLINI, S.; ARAKI, C. A. S.; SILVA, A. C. M.; NAKAGAWA, J. Teste de comprimento de plântula na avaliação da qualidade fisiológica de sementes de soja. Revista Brasileira de Sementes, v.29, n.2, p.90-96, 2007. 